\title{
Topological Insulators and Superconductors from String Theory
}

\author{
Shinsei Ryu \\ Department of Physics, University of California, Berkeley, CA 94720, USA \\ Tadashi Takayanagi \\ Institute for the Physics and Mathematics of the Universe (IPMU), \\ University of Tokyo, Kashiwa, Chiba 277-8582, Japan
}

(Dated: October 24, 2018)

\begin{abstract}
Topological insulators and superconductors in different spatial dimensions and with different discrete symmetries have been fully classified recently, revealing a periodic structure for the pattern of possible types of topological insulators and supercondutors, both in terms of spatial dimensions and in terms of symmetry classes. It was proposed that K-theory is behind the periodicity. On the other hand, D-branes, a solitonic object in string theory, are also known to be classified by K-theory. In this paper, by inspecting low-energy effective field theories realized by two parallel D-branes, we establish a one-to-one correspondence between the K-theory classification of topological insulators/superconductors and D-brane charges. In addition, the string theory realization of topological insulators and superconductors comes naturally with gauge interactions, and the WessZumino term of the D-branes gives rise to a gauge field theory of topological nature, such as ones with the Chern-Simons term or the $\theta$-term in various dimensions. This sheds light on topological insulators and superconductors beyond non-interacting systems, and the underlying topological field theory description thereof. In particular, our string theory realization includes the honeycomb lattice Kitaev model in two spatial dimensions, and its higher-dimensional extensions. Increasing the number of D-branes naturally leads to a realization of topological insulators and superconductors in terms of holography (AdS/CFT).
\end{abstract}

PACS numbers: 72.10.-d,73.21.-b,73.50.Fq

\section{INTRODUCTION}

The integer quantum Hall effect (IQHE) is one of the most striking phenomenon in the $d=2$-dimensional electron system under a strong magnetic field, and has been one of the central topics in condensed matter physics. As well-known, the Hall conductance is quantized when the electronic ground state has a non-trivial topological structure.

With the recent discovery of the quantum spin Hall effect (QSHE) in $d=2$ and the $\mathbb{Z}_{2}$ topological insulator in $d=3$ [1], it has become clear that topological phases can exist in a much wider context, i.e., in the spatial dimension other then $d=2$, and without strong timereversal symmetry (TRS) breaking by a magnetic field. The QSHE and $d=3$-dimensional $\mathbb{Z}_{2}$ topological insulator can be thought of as a close cousin of the IQHE, but different from the IQHE in many essential ways: these states can exist only when time-reversal symmetry is respected, and can be either two- or three-dimensional. Furthermore, $\mathbb{Z}_{2}$ topological insulators are characterized by a binary topological number, unlike the integral Hall conductivity in the IQHE. Recent experiments confirmed HgTe quantum well and Bismuth-related materials, both of which have strong spin-orbit coupling, indeed realize such non-trivial $\mathbb{Z}_{2}$ topological phases.

The Bloch wavefunctions in the IQHE or the $\mathbb{Z}_{2}$ topological insulator in $d=2$ (the QSHE) and in $d=3$ have non-trivial topology, detected by an integer or a binary topological number. For superconductors (superfluid), at least within the BCS meanfield theory, it is possible the wavefunctions of fermionic Bogoliubov quasiparticles carry non-trivial topological characters, very much the same way as the electronic wavefunctions in the IQHE or the $\mathbb{Z}_{2}$ topological insulator, in particular when fully gapped (i.e., a quasiparticle gap opens for entire momentum space); such superconductors (superfluid) can be called topological superconductor (superfluid). A wellknown example of such topological superconductor is the $d=2$-dimensional chiral $p$-wave superconductor which has a $p_{x}+i p_{y}$-wave superconducting order parameter.

For non-interacting fermions, an exhaustive classification of topological insulators (TIs) and superconductors (TSCs) is proposed in Refs. 15, 16]: TIs/TSCs are classified in terms of spatial dimensions $d$ and the $10=2+8$ symmetry classes (two "complex" and eight "real" classes), each characterized by presence/absence of discrete symmetries such as time-reversal symmetry (TRS or T), particle-hole symmetry (PHS or C), and chiral (or sublattice) symmetry (SLS or S). (Table fi). In relativistic field theories, the first two are the same as the usual $\mathrm{T}$ and $\mathrm{C}$ symmetries. For any system which has both $\mathrm{T}$ and $\mathrm{C}$ symmetries, $\mathrm{S}$ symmetry (SLS) is realized as a product $\mathrm{C} \cdot \mathrm{T}$, while it can exist on its own, even without $\mathrm{T}$ and $\mathrm{C}$ symmetries. The ten symmetry classes are in one-to-one correspondence to the Riemannian symmetric spaces without exceptional series (described in Table II) [18 21, and to K-theory classifying spaces [16, 17]. For example, the integer QHE, QSHE, and $\mathbb{Z}_{2}$ TI are a topologically non-trivial state belonging to class A $(d=2)$, AII $(d=2)$, and AII $(d=3)$, respectively. 
The classification reveals a periodicity both in spatial dimensions $d$ and in symmetry classes, and hence is often called "periodic table" of TIs/TSCs. Not only it incorporate many of previously known topological phases, it also predicted new topological phases; e.g., the B phase of ${ }^{3} \mathrm{He}$ was newly identified as a topological phase; the existence of the $d=3$-dimensional topological singlet superconductor was predicted, and verified by an explicit construction of a lattice model of the BCS superconductor 22 .

The complete classification of non-interacting TIs and TSCs opens up a number of further questions, most interesting among which are interaction effects 23: Do non-interacting topological phases continue to exist in the presence of interactions? Can interactions give rise to novel topological phases other than non-interacting TIs/TSCs? What is a topological field theory underlying TIs/TSCs, which can potentially describe TIs/TSCs beyond non-interacting examples?, etc.

On the other hand, the ten-fold classification of TIs/TSCs reminds us of D-branes, which are fundamental objects in string theory, and are also classified by K-theory 24, 25 (Table III) via the open string tachyon condensation [26, 27]. It is then natural to speculate a possible connection between TIs/TSCs and of D-branes [28. In this paper, we propose a systematic construction of TIs/TSCs in terms of various systems composed of two kinds of D-branes ( $\mathrm{D} p$ - and $\mathrm{D} q$-branes), possibly with an orientifold plane (O-plane). Besides the appealing mathematical similarity between TIs/TSCs and D-branes, realizing TIs/TSCs in string theory has a number of merits, since string theory and D-branes are believed to be rich enough to reproduce many types of field theories and interactions in a fully consistent and UV complete way. Indeed, our string theory realizations of TIs/TSCs give rise to massive fermion spectra, which are in one-to-one correspondence with the ten-fold classification of TIs/TSCs, and come quite naturally with gauge interactions. These systems, while interacting, are all topologically stable, as protected by the K-theory charge of D-branes. We thus make a first step toward understanding interacting TIs/TSCs. Our approach also reveals the connection between the number of symmetry classes of TIs/TSCs and the critical dimension of superstring theory $(=10)$, via the Bott periodicity of K-theory.

In $\mathrm{D} p$ - $\mathrm{D} q$-systems, massive fermions arise as an open string excitation between the two D-branes. The distance between the branes corresponds to the mass of fermions. Open strings ending on the same D-branes give rise to a gauge field, which we call $A_{\mu}(\mathrm{D} p)$ and $\tilde{A}_{\mu}(\mathrm{D} q)$ with gauge group $G$ and $\tilde{G}$, respectively, and couple to the fermions (they are identified as in Table IV). These two gauge fields play different roles in our construction: The gauge field $A_{\mu}$ "measures" the K-theory charge of the $\mathrm{D} q$ brane, and in that sense it can be interpreted as an "external" gauge field. In this picture, the D $q$-brane charge is identified with the topological (K-theory) charge of TIs/TSCs. On the other hand, $\tilde{A}_{\mu}$ is an internal degree of freedom on the Dq-brane.

The massive fermions can be integrated out, yielding the description of the topological phase in terms of the gauge fields. The resulting effective field theory comes with terms of topological nature, such as the ChernSimons (CS) or the $\theta$-terms. In our string theory setup, they can be read off from the Wess-Zumino (WZ) action of the D-branes, by taking one of the D-branes as a background for the other. In our construction of the QSHE (i.e. AII $d=2$ ), the brane system consists of a $\mathrm{D} p$-brane and a $\mathrm{D} q-\overline{\mathrm{D}} q$ system, where $\overline{\mathrm{D}} q$ denotes an anti Dq-brane, which has the opposite Ramond-Ramond charge to the $\mathrm{D} q$-brane. The $\mathrm{D} p$-brane has an $\mathrm{SU}(2)$ gauge field and the $\mathrm{D} q-\overline{\mathrm{D}} q$ system has a $\mathrm{U}(1)$ gauge field. The former couples to the $\mathrm{SU}(2)$ spin rotation, while the latter is the usual electric-magnetic field. The integration of massive fermions between the $\mathrm{D} p$-brane and $\mathrm{D} q-\overline{\mathrm{D}} q$ system produces the double Chern-Simons coupling, which agrees with the previously proposed description of quantum spin Hall effect. One can view these gauge-interacting TIs/TSCs from $\mathrm{D} p$-D $q$-systems as an analogue of the projective (parton) construction of the (fractional) QHE 29.

Our string theory realization of TIs/TSCs sheds light on extending the projective construction of the QHE to more generic TIs/TSCs; it tells us what type of gauge field is "natural" to couple with fermions in topological phases, and guarantees the topological stability of the system.

This paper is organized as follows. In Sec. II, we will present our D-brane construction of TIs/TSCs for two "complex" symmetry classes A and AIII, which correspond to the complex K-group. Expecting readers from both high-energy and condensed matter communities, the underlying principles of the construction are summarized in Sec. II A, together with a brief but pedagogical review of D-branes and open strings. In Sec. III], we will give D-brane constructions for remaining eight classes which are classified by the real K-group. This will be followed by a detailed comparison between the Dbrane system and topological phases in condensed matter physics in Sec. IIIF. In section IV, we will draw conclusions and discuss future problems. In Appendix A, we gave a brief explanation on the open string spectrum in the presence of D-branes and orientifolds. This paper is an extend version of our previous brief report [30].

\section{D-BRANE CONSTRUCTION FOR COMPLEX CASE}

\section{A. Basic Strategy}

1. open string and D-branes

To realize a TI/TSC in string theory, we need massive fermions, which are charged under some gauge field. One of the simplest setups in string theory which gives rise to 


\begin{tabular}{c|cccccccc|ccc}
\hline \hline class $\backslash d$ & 0 & 1 & 2 & 3 & 4 & 5 & 6 & 7 & $\mathrm{~T}$ & $\mathrm{C}$ & $\mathrm{S}$ \\
\hline $\mathrm{A}$ & $\mathbb{Z}$ & 0 & $\mathbb{Z}$ & 0 & $\mathbb{Z}$ & 0 & $\mathbb{Z}$ & 0 & 0 & 0 & 0 \\
AIII & 0 & $\mathbb{Z}$ & 0 & $\mathbb{Z}$ & 0 & $\mathbb{Z}$ & 0 & $\mathbb{Z}$ & 0 & 0 & 1 \\
\hline AI & $\mathbb{Z}$ & 0 & 0 & 0 & $2 \mathbb{Z}$ & 0 & $\mathbb{Z}_{2}$ & $\mathbb{Z}_{2}$ & + & 0 & 0 \\
BDI & $\mathbb{Z}_{2}$ & $\mathbb{Z}$ & 0 & 0 & 0 & $2 \mathbb{Z}$ & 0 & $\mathbb{Z}_{2}$ & + & + & 1 \\
D & $\mathbb{Z}_{2}$ & $\mathbb{Z}_{2}$ & $\mathbb{Z}$ & 0 & 0 & 0 & $2 \mathbb{Z}$ & 0 & 0 & + & 0 \\
DIII & 0 & $\mathbb{Z}_{2}$ & $\mathbb{Z}_{2}$ & $\mathbb{Z}$ & 0 & 0 & 0 & $2 \mathbb{Z}$ & - & + & 1 \\
AII & $2 \mathbb{Z}$ & 0 & $\mathbb{Z}_{2}$ & $\mathbb{Z}_{2}$ & $\mathbb{Z}$ & 0 & 0 & 0 & - & 0 & 0 \\
CII & 0 & $2 \mathbb{Z}$ & 0 & $\mathbb{Z}_{2}$ & $\mathbb{Z}_{2}$ & $\mathbb{Z}$ & 0 & 0 & - & - & 1 \\
C & 0 & 0 & $2 \mathbb{Z}_{0}$ & $\mathbb{Z}_{2}$ & $\mathbb{Z}_{2}$ & $\mathbb{Z}$ & 0 & 0 & - & 0 \\
CI & 0 & 0 & 0 & $2 \mathbb{Z}$ & 0 & $\mathbb{Z}_{2}$ & $\mathbb{Z}_{2}$ & $\mathbb{Z}$ & + & - & 1 \\
\hline \hline
\end{tabular}

TABLE I: Classification of topological insulators and superconductors 15, 16]; $d$ is the space dimension; the left-most column (A, AIII, ..., CI) denotes the ten symmetry classes of fermionic Hamiltonians, which are characterized by the presence/absence of time-reversal (T), particle-hole (C), and chiral (or sublattice) (S) symmetries of different types denoted by \pm 1 in the right most three columns. The entries " $\mathbb{Z}$ ", " $\mathbb{Z}_{2}$ ", " $2 \mathbb{Z}$ ", and " 0 " represent the presence/absence of topological insulators and superconductors, and when they exist, types of these states (see Ref. [15] for detailed descriptions).

\begin{tabular}{cc}
\hline \hline Cartan label & Riemannian Symmetric Spaces \\
\hline \hline A & $\mathrm{U}(N) \times \mathrm{U}(N) / \mathrm{U}(N)$ \\
AII & $\mathrm{U}(N+M) / \mathrm{U}(N) \times \mathrm{U}(M)$ \\
\hline \hline AI & $\mathrm{U}(N) / \mathrm{O}(N)$ \\
BDI & $\mathrm{O}(N+M) / \mathrm{O}(N) \times \mathrm{O}(M)$ \\
D & $\mathrm{O}(N) \times \mathrm{O}(N) / \mathrm{O}(N)$ \\
DIII & $\mathrm{SO}(2 N) / \mathrm{U}(N)$ \\
AII & $\mathrm{U}(2 N) / \mathrm{Sp}(2 N)$ \\
CII & $\mathrm{Sp}(N+M) / \mathrm{Sp}(N) \times \mathrm{Sp}(M)$ \\
C & $\mathrm{Sp}(2 N) \times \mathrm{Sp}(2 N) / \mathrm{Sp}(2 N)$ \\
CI & $\mathrm{Sp}(2 N) / \mathrm{U}(N)$ \\
\hline \hline
\end{tabular}

TABLE II: Cartan classification of Riemannian symmetric spaces 21.

charged massive fermions is a system that consists of two kinds of D-branes: $\mathrm{D} p$ - and $\mathrm{D} q$-branes.

A D $p$-brane is a solitonic object in string theory whose world volume is $(p+1)$-dimensional. For our purposes, a $\mathrm{D} p$-brane can simply be regarded as a $(p+1)$-dimensional

\begin{tabular}{cccccccccccc}
\hline \hline & $\mathrm{D}(-1)$ & D0 & D1 & D2 & D3 & D4 & D5 & D6 & D7 & D8 & D9 \\
\hline type IIB & $\mathbb{Z}$ & 0 & $\mathbb{Z}$ & 0 & $\mathbb{Z}$ & 0 & $\mathbb{Z}$ & 0 & $\mathbb{Z}$ & 0 & $\mathbb{Z}$ \\
O9 $^{-}$(type I) & $\mathbb{Z}_{2}$ & $\mathbb{Z}_{2}$ & $\mathbb{Z}$ & 0 & 0 & 0 & $\mathbb{Z}$ & 0 & $\mathbb{Z}_{2}$ & $\mathbb{Z}_{2}$ & $\mathbb{Z}$ \\
O9 $^{+}$ & 0 & 0 & $\mathbb{Z}$ & 0 & $\mathbb{Z}_{2}$ & $\mathbb{Z}_{2}$ & $\mathbb{Z}$ & 0 & 0 & 0 & $\mathbb{Z}$ \\
\hline \hline
\end{tabular}

TABLE III: $\mathrm{D} p$-brane charges from K-theory, classified by $\mathrm{K}\left(\mathbb{S}^{9-p}\right), \mathrm{KO}\left(\mathbb{S}^{9-p}\right)$ and $\mathrm{KSp}\left(\mathbb{S}^{9-p}\right)$ 24]. A $\mathbb{Z}_{2}$ charged $\mathrm{D} p$ brane with $p$ even or $p$ odd represents a non-BPS D $p$-brane or a bound state of a $\mathrm{D} p$ and an anti- $\mathrm{D} p$ brane, respectively [26].

\begin{tabular}{c|c|cccccccc}
\hline \hline$G$ & class $\backslash d$ & 0 & 1 & 2 & 3 & 4 & 5 & 6 & 7 \\
\hline $\mathrm{U}$ & $\mathrm{A}$ & $\mathrm{U}$ & - & $\mathrm{U}$ & - & $\mathrm{U}$ & - & $\mathrm{U}$ & - \\
$\mathrm{U}$ & $\mathrm{AIII}$ & - & $\mathrm{U}$ & - & $\mathrm{U}$ & - & $\mathrm{U}$ & - & $\mathrm{U}$ \\
\hline $\mathrm{O}$ & $\mathrm{AI}$ & $\mathrm{O}$ & - & - & - & $\mathrm{Sp}$ & - & $\mathrm{U}$ & $\mathrm{O}$ \\
$\mathrm{O}$ & $\mathrm{BDI}$ & $\mathrm{O}$ & $\mathrm{O}$ & - & - & - & $\mathrm{Sp}$ & - & $\mathrm{U}$ \\
$\mathrm{O}$ & $\mathrm{D}$ & $\mathrm{U}$ & $\mathrm{O}$ & $\mathrm{O}$ & - & - & - & $\mathrm{Sp}$ & - \\
$\mathrm{O}$ & $\mathrm{DIII}$ & - & $\mathrm{U}$ & $\mathrm{O}$ & $\mathrm{O}$ & - & - & - & $\mathrm{Sp}$ \\
$\mathrm{Sp}$ & $\mathrm{AII}$ & $\mathrm{Sp}$ & - & $\mathrm{U}$ & $\mathrm{O}$ & $\mathrm{O}$ & - & - & - \\
$\mathrm{Sp}$ & $\mathrm{CII}$ & - & $\mathrm{Sp}$ & - & $\mathrm{U}$ & $\mathrm{O}$ & $\mathrm{O}$ & - & - \\
$\mathrm{Sp}$ & $\mathrm{C}$ & - & - & $\mathrm{Sp}$ & - & $\mathrm{U}$ & $\mathrm{O}$ & $\mathrm{O}$ & - \\
$\mathrm{Sp}$ & $\mathrm{CI}$ & - & - & - & $\mathrm{Sp}$ & - & $\mathrm{U}$ & $\mathrm{O}$ & $\mathrm{O}$ \\
\hline \hline
\end{tabular}

TABLE IV: External $G$ (left-most column) and internal $\tilde{G}$ gauge groups for each spatial dimension $d$ and symmetry class; $\mathrm{U}, \mathrm{O}$, Sp, represents $\mathrm{U}(1), \mathrm{O}(1)=\mathbb{Z}_{2}$, and $\mathrm{Sp}(1)=\mathrm{SU}(2)$, respectively.

plane (for more details refer to, for example, Ref. [31]). An open string can ends at a D-brane, i.e., a D-brane is defined as the set of end points of open strings. In other words, an open string attached to a D $p$-brane satisfies the Neumann boundary condition in the $(p+1)$ directions parallel with the D-brane, while it satisfies the Dirichlet boundary condition in the remaining $(9-p)$ directions orthogonal to the D-brane.

An open string has two end points, and in the presence of $N$ coincident $\mathrm{D} p$-branes, say, these two ends can be attached to any one of the $N \mathrm{D} p$-branes. Open string states can thus be labeled, in addition to momentum and Fock-space states labeling the internal degrees of freedom of the string, by an entry of an $N \times N$ matrix (the ChanPaton matrix). Indeed we can show a $(p+1)$-dimensional $\mathrm{U}(N)$ gauge theory lives on the $N$ coincident $\mathrm{D} p$-branes. In our construction, the gauge field on the $\mathrm{D} p$-brane and D $q$-brane are denoted by $A_{\mu}$ and $\tilde{A}_{\mu}$, which are called the external and internal gauge field, respectively. See (a) in Fig.1.

Open string excitations between the $\mathrm{D} p$ - and $\mathrm{D} q$ branes give rise to fermions. (In addition, scalar bosons, which is not our main focus in this paper, appear sometime as we explain below.) The mass $m$ of these fermions are proportional to the distance $\Delta x$ between the $\mathrm{D} p$ - and $\mathrm{D} q$-branes, given by $m=T_{\text {string }} \Delta x$, where $T_{\text {string }}$ is the string tension (often denoted by $T_{\text {string }} \equiv 1 /\left(2 \pi \alpha^{\prime}\right)$ ). We define the common dimension of the $\mathrm{D} p$ - and $\mathrm{D} q$-branes to be $d+1$ and the TIs/TSCs in our construction live in the $d+1$ dimensions because the massive fermions are confined in this directions.

One of the reasons why our strategy to construct topological phases is based on realizing charged massive fermions is that, in the presence of a certain defect (boundary), quite generically, such fermions give rise to modes localized at the defect (boundary) - a defining property of TIs/TSCs. (In reality, the appearance of such a boundary is to some extent inevitable when we attach probes to measure various physical quantities.) These points will further be elaborated in Sec. IID. 


\section{Neumann-Neumann, Dirichlet-Dirichlet, and Neumann-Dirichlet directions}

The most important characteristics of such D-brane systems are the boundary conditions of open strings stretching between the $\mathrm{D} p$ - and $\mathrm{D} q$-branes, as they are the origin of the charged fermions. Since an open string has two end points each of which satisfies either the Neumann (N) or Dirichlet (D) boundary condition, we distinguish three possibilities, i.e., (i) both ends satisfy the $\mathrm{N}$ boundary condition (NN), (ii) both ends satisfy the D boundary condition (DD), and (iii) mixed boundary condition where one end satisfies the $\mathrm{N}$ boundary condition while the other satisfies the $\mathrm{D}$ boundary condition (ND). The number of these directions, denoted by \#NN, \#DD and \#ND, are completely fixed once we determine the directions in which the D-branes extend. Since the total spacetime dimensions of superstring (called critical dimension) is ten, they are subjected to the constraint,

$$
\# \mathrm{NN}+\# \mathrm{DD}+\# \mathrm{ND}=10 .
$$

In our construction, \#NN and \#DD have a physical meaning; \#NN is equal to the spacetime dimensions of TIs/TSCs, \#NN $=d+1$, while \#DD represents the number of possible mass deformations (types of mass perturbations which can be added to the charge fermion system).

In string theory, there is a very important symmetry called T-duality. If we take a T-duality in a NN or DD direction, the dimension $d$ decreases or increases since T-duality turns a NN (DD) direction into a DD (NN) direction, respectively. At the same time, type IIA and IIB string theory are exchanged with each other. On the other hand, T-duality in a ND direction separately preserves \#NN, \#DD and \#ND. This does not change the dimensions $d$ of TIs/TSCs, while $p$ and $q$ are shifted. Therefore, this degree of freedom, taking T-duality in ND directions, can be thought as somewhat redundant for our purposes, and we can make use of it to fix the value of $p$.

Once we fix $p$ (we choose $p=5$ for the most part of this section), each brane system is completely classified by the two integers \#NN $=d+1$ and \#DD. They encode the information how the $\mathrm{D} q$-brane is embedding into the ten dimensional spacetime. In this picture, the D $q$-brane charge is identified with the topological charges of the TIs/TSCs. Indeed, both are classified by K-theory.

Properties of the system realized in the string spectrum for an open string stretching between the $\mathrm{D} p$ - and $\mathrm{D} q$ branes depend sensitively on \#NN, \#DD and \#ND. For example, when \#ND $\leq 4$, the $\mathrm{D} p$-D $q$ open string includes scalar bosons in addition to a massive fermion. Moreover, when $\# \mathrm{ND} \leq 3$, they become tachyons which makes the brane system unstable; this constraints the possible values of \#NN, \#DD and \#ND to realize TIs/TSCs.

Furthermore, we will focus on the cases with \#DD = 1,2 , as these cases lead to a stable gapless mode, once we introduce a boundary to the system. Studying the open string spectrum between the $\mathrm{D} p$ - and $\mathrm{D} q$-branes for $\# \mathrm{DD} \geq 3$, we find a stable (=tachyon free) configuration if $\# \mathrm{DD} \leq 5-d$. Thus it is possible to consider brane systems with \#DD $=3,4$ in order to realize TIs/TSCs in $d=1,2$. However, since these examples have more \#DD directions as compared with the realizations with $\# \mathrm{DD}=1,2$, they can simply be regarded as multiple copies of class A or AIII TIs/TSCs. In other words, these new configurations have the non-abelian global symmetry given by $\mathrm{SO}(\# \mathrm{DD})$, and if we mod out the system by this symmetry, it reduces to the previous cases. Therefore we will not discuss examples with $\#$ DD $\geq 3$ in this paper.

The strategy explained above is enough to identify Dbrane configurations for the class A and AIII, which belong to the complex category in a K-theoretic sense. To find D-brane systems for other eight classes, which correspond to the real category, we need another object called orientifolds (see (b) in Fig.11), which will be discussed separately in the next section.

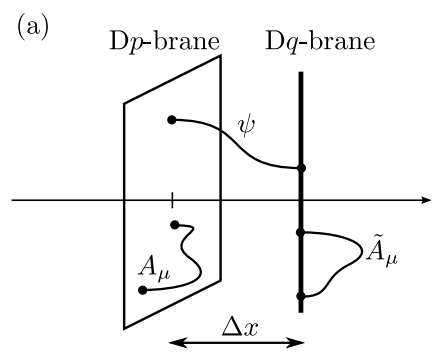

(c)
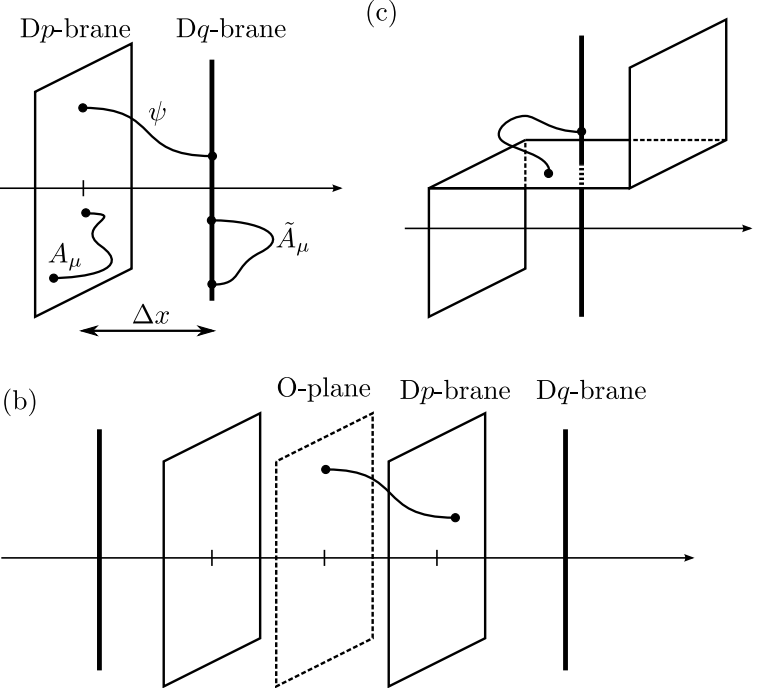

FIG. 1: (a) The $\mathrm{D} p-\mathrm{D} q$ system realizing TIs/TSCs in the complex case. (b) The $\mathrm{D} p$-D $q$ system with O-plane realizing TIs/TSCs in the real case. (c) The intersecting $\mathrm{D} p$-D $q$ system realizing a boundary in TIs/TSCs.

\section{B. Class A}

describe the D-brane construction of class A TIs. In the D-brane construction outlined above, the system is composed of a D5- and D $q$-brane ( $p$ is fixed to be $p=5$ by making use of T-duality). As mentioned earlier, we require that the number of mass deformations is one, \#DD $=1$, since TIs/TSCs should by definition have gapless modes at the boundary (edge, surface, etc.). In our D-brane construction, as we will come back later in more detail in the section IID, putting a boundary in a TI/TSC is realized by bending the $\mathrm{D} p(=\mathrm{D} 5)$-brane at 
the boundary so that it intersects with the $\mathrm{D} q$-brane in an orthogonal way, as depicted in Fig. I (c). Massless modes appear only if the bent D5-brane intersects with $\mathrm{D} q$-brane along the $d-1$ dimensional boundary. This is only possible when \#DD = 1 under generic situations.

In this case, we can take a D5-brane in type IIB string theory which extends in the $x^{0,1,2,3,4,5}$ directions in ten-dimensional space-time without losing any generality. The massive fermions live on the $d=q-3$ spacial dimensions which are common to the D5- and D $q$-branes. We identify these configurations with the class A TIs in spatial dimensions $d=0,2,4$, as summarized in Table V. Indeed this is consistent with the known topological charge in Table 1 .

Among these, the most familiar example is the QHE (class $\mathrm{A}$ in $d=2$ ). In our construction, this is realized as a D5-D5 system. By T-duality, this setup is equivalent to the D3-D7 system studied in Refs. 32 34]. Since \#ND = 6 , open string excitations between the D5-branes give rise to two Majorana fermions $(\mathrm{Mj})$ or equivalently one two-component Dirac fermion (Di), $\psi$, and no bosons. For details of counting of fermions, refer to the appendix A. The distance between the D-branes in $x^{9}$ direction $\left(\Delta x^{9}\right)$ is proportional to the mass $m$ of the fermions. The massive fermion is interpreted as an electron and it couples to an electric-magnetic field $A_{\mu}$, which is the $\mathrm{U}(1)$ gauge field on the $\mathrm{D} p(=\mathrm{D} 5)$-brane.

The low-energy effective theory is schematically summarized by the effective Lagrangian in the $(2+1)$ dimensional common direction of the two D5-branes,

$$
\mathcal{L}=\bar{\psi}\left[\gamma^{\mu}\left(i \partial_{\mu}-A_{\mu}-\tilde{A}_{\mu}\right)-m\right] \psi+\cdots,
$$

where the gamma matrices are given, in terms of the $2 \times 2$ Pauli matrices, by $\gamma^{\mu=0,1,2}=\sigma_{3}, i \sigma_{2},-i \sigma_{1}$, say. (We will use $\sigma_{0}$ to denote the $2 \times 2$ identity matrix.)

Integrating out the massive fermions yields the CS term

$$
S_{\mathrm{CS}}=\frac{k}{8 \pi} \int A \wedge d A, \quad k=\frac{m}{|m|}= \pm 1
$$

where we set $\tilde{A}=0$ for simplicity. The Hall conductivity is read off from the CS term for $A_{\mu}$ as

$$
\sigma_{x y}=\frac{k}{4 \pi} \text {. }
$$

When we change the sign of $m$ by passing the $\mathrm{D} q$-brane through the $\mathrm{D} p$-brane, the value of $k$ jumps from -1 to +1 . If we instead put $N_{f} \mathrm{D} q$-branes, we have $N_{f}$ copies of massive Dirac fermions $\psi_{i}$ which couple with U(1) photon field $A_{\mu}$ and $\mathrm{U}\left(N_{f}\right)$ gauge fields $\tilde{A}_{\mu}$ (when all $\mathrm{D} q$-branes are coincident), leading to the Hall conductivity $\sigma_{x y}=$ $N_{f} k /(4 \pi)$.

It is indeed possible to derive the CS term (3) from the Wess-Zumino (WZ) term of the D5-brane, which expresses how D-branes couple to various Ramond-Ramond (RR) fields. Notice that the low energy effective theory of a D-brane is given by the sum of the Dirac-Born-Infeld action and the WZ term. The former is a non-linear analogue of the Yang-Mills action and is irrelevant in our topological argument below. Since this $d=2$ example can be naturally generalized to the $d=0,4$ cases, below we consider the WZ term of the D5-brane for general dimensions $d$ :

$$
S_{\mathrm{WZ}}=\mu_{\mathrm{D} 5} \int_{\mathrm{D} 5} C_{4-d} \wedge e^{2 \pi \alpha^{\prime} F},
$$

where $\mu_{\mathrm{D} 5}$ is the $\mathrm{RR}$ charge of the $\mathrm{D} 5$-brane and $C_{n}$ is the RR $n$-form potential and $F=d A$ is the field strength of the gauge field on the D5-brane. This is evaluated in the presence of the $\mathrm{D} q$-brane as follows

$$
S_{\mathrm{WZ}}= \pm \frac{1}{2(2 \pi)^{d / 2}(1+d / 2) !} \int_{\mathbb{R}^{1, d}} \operatorname{Tr}\left[A \wedge F^{\frac{d}{2}}\right],
$$

where we noted the fact that a $\mathrm{D} q$-brane induces a half of the unit RR flux $2 \pi \int_{S^{5-d}} d C_{5-d}= \pm 1$ since in our setup the $\mathrm{D} p$-brane is located on either one of the two sides of the $\mathrm{D} q$-brane, but it does not enclose the $\mathrm{D} q$ brane completely. The sign in Eq. (6) depends on which side of the Dq-brane the D5-brane is situated. If we set $d=2$ in Eq. (6), we obtain $\pm \frac{1}{8 \pi} \int A \wedge F$ and this agrees with (3). Notice also that a topological insulator with the topological charge $w \in \mathbb{Z}$ is given by the one with $w$ copies of $\mathrm{D} q$-branes and thus has the topological term given by $w$ times (6).
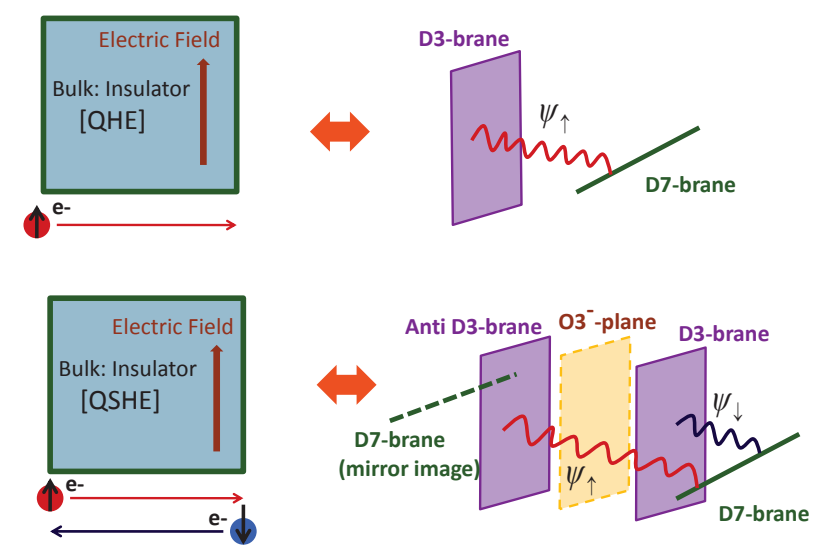

FIG. 2: Schematic description of the QHE (up) and the QSHE (down) and their brane configurations (right) For the presentational convenience, we took the T-duals of our previous brane setups. Refer to Table. [X].

\section{Class AIII}

Now let us turn to class AIII, which is characterized by the presence of an extra symmetry, SLS. We argue that 
Class A:

\begin{tabular}{c|cccccc|cccc|c|c}
\hline & 0 & 1 & 2 & 3 & 4 & 5 & 6 & 7 & 8 & 9 & $d$ & $\mathrm{~A}$ \\
\hline D5 & $\times$ & $\times$ & $\times$ & $\times$ & $\times$ & $\times$ & & & & & \\
\hline D3 & $\times$ & & & & & & $\times$ & $\times$ & $\times$ & 0 & $\mathbb{Z}(2 \mathrm{Mj})$ \\
D5 & $\times$ & $\times$ & $\times$ & & & & $\times$ & $\times$ & $\times$ & 2 & $\mathbb{Z}(2 \mathrm{Mj})$ \\
D7 & $\times$ & $\times$ & $\times$ & $\times$ & $\times$ & & $\times$ & $\times$ & $\times$ & 4 & $\mathbb{Z}(1 \mathrm{Di})$ \\
\hline \hline
\end{tabular}

Class AIII:

\begin{tabular}{c|cccccc|cccc|c|c}
\hline \hline & 0 & 1 & 2 & 3 & 4 & 5 & 6 & 7 & 8 & 9 & $d$ & AIII \\
\hline D4 & $\times$ & & $\times$ & $\times$ & $\times$ & $\times$ & & & & & & \\
\hline $\mathrm{D} 4$ & $\times$ & & $\times$ & & & & $\times$ & $\times$ & $\times$ & & 1 & $\mathbb{Z}(2 \mathrm{Mj})$ \\
$\mathrm{D} 6$ & $\times$ & & $\times$ & $\times$ & $\times$ & & $\times$ & $\times$ & $\times$ & & 3 & $\mathbb{Z}(2 \mathrm{Mj})$ \\
\hline \hline
\end{tabular}

TABLE V: $\mathrm{D} p$-D $q$ systems for class A and AIII where $p=5$ and $q=3,5,7$ for A, and $p=4$ and $q=4,6$ for AIII. The D-branes extend in the $\mu$-th direction denoted by " $\times$ " in the ten-dimensional space-time $(\mu=0, \ldots, 9) ; d+1$ is the number of common directions of $\mathrm{D} p$-and $\mathrm{D} q$-branes; The last column shows the Dq-brane charge, together with fermion spectra per Dq-brane, where " $N_{f} \mathrm{Mj}$ " or " $N_{f}$ Di" represents $N_{f}$ flavor of Majorana and Dirac spinors, respectively [37.

SLS is equivalent to an invariance of the brane configurations under inversion of a coordinate in the DirichletDirichlet (DD) directions of open strings between the $\mathrm{D} p$ and $\mathrm{D} q$-branes. The existence of boundary gapless modes is guaranteed only if we assume $\# \mathrm{DD}=2$. If we take $x^{1}$ and $x^{9}$-directions as the two DD directions, SLS requires $x^{1}=0$, which guarantees the $\mathrm{D} p$ - and $\mathrm{D} q$-branes intersect when the $\mathrm{D} p$-brane is bent toward the $\mathrm{D} q$-brane.

Indeed such a configuration is obtained by taking Tdual of class A configurations in $x_{1}$-direction (Table $\mathrm{V}$ ). Again, the fermion spectrum consists of two Majorana fermions (=one Dirac fermion) for all dimensions, and the mass of the fermion is, again, proportional to $\Delta x^{9}$. This prescription completely agrees with what is expected from the Dirac fermion representatives of class AIII TIs in Ref. [15]; The $d$-dimensional class AIII system can be regarded as a dimensional reduction from the $(d+1)$-dimensional class $\mathrm{A}$ system by setting one of the components of the momentum to be zero.

In class AIII TIs/TSCs, the integral topological charge is related to the coefficient of the topological term $\int F^{(d+1) / 2}$. The coefficient gets shift by one unit by sign flip $m \rightarrow-m$ of the fermion mass $m$. Consider, as an example, the $d=3$ case and assume that there are $N_{f}$ D6-branes. By integrating out the $N_{f}$ massive Dirac fermions, we find the following topological term for the $\mathrm{U}(1)$ field strength 36.

$$
\frac{m}{16 \pi^{2}|m|} N_{f} \pi \int_{\mathbb{R}^{1,3}} F \wedge F
$$

Since we do not assume any time reversal symmetry in this class, we can take $\theta$ any constant. Its physical consequence is that if the system has a boundary, there exist $n$ massless fermions on its boundary.

\section{Boundary Gapless Modes of TIs/TSCs}

A defining property of TIs/TSCs is the appearance of stable gapless degrees of freedom, when the system is terminated by a $(d-1)$-dimensional boundary. The bestknown example is the $(1+1)$-dimensional edge state in the QHE, i.e. class $\mathrm{A}$ in $d=2$. The integer quantum Hall edge state is a chiral (Weyl) fermion, and always conducting even in the presence of strong disorder, as far as the bulk topological character is not destroyed by disorder.

Quite generally, we can model a boundary in TIs/TSCs by considering a position dependent fermion mass term. Let us consider a mass $m=m(y)$ which changes along one spatial direction, $y$-direction, say, and changes its sign at $y=0, m(y)>0$ for $y<0, m(y)<0$ for $y>0$, and $m(y)=0$ at $y=0$. I.e., the mass function $m(y)$ has a kink at the boundary such as $m(y) \propto y$ when $y$ is very small, while $m(y)$ is almost a constant in the bulk region.

Such kink or domain wall defines a $(d-1)$-dimensional boundary (or interface) which is located at $y=0$ and is flat (straight) along the $(d-1)$ directions. The band gap vanishes locally at the boundary $m(y=0)=0$. As well known in the domain wall fermion or in the index theorems of various forms, such topological defect (kink) induces a fermion zero mode localized at the defect, which in our context is a gapless fermion mode signaling a topological character of the bulk.

In our brane construction, the sample boundary can be constructed by "bending" the D $p$-brane toward the $\mathrm{D} q$-brane, to create an intersection between these branes (Fig. 11). This leads to a position-dependent fermion mass, which changes its sign at the intersection as we wanted. This increases \#ND by two and the correct number of massless fermions appears at the intersection.

For example, let us consider the boundary modes for class A and AIII. By bending the D p-brane world-volume in Table $\mathrm{V}$ as we mentioned, we find the D-brane configurations with boundary as described in Table VI. If we look at class $\mathrm{A}$ at $d=1$, then we find a Weyl fermion and this corresponds to the chiral fermion of the edge state in the QHE.

\section{D-BRANE CONSTRUCTION FOR REAL CASE}

To realize other eight "real" symmetry classes in string theory, we need to implement either one of TRS or PHS, or both. To respect PHS, which is equivalent to charge conjugation invariance, we require the gauge fields $A_{\mu}$ and $\tilde{A}_{\mu}$ are not independent of their complex conjugates. This is the same as the orientation $(\Omega)$ projection of gauge theories on D-branes in string theory.

To realize TRS, we recall that it can be viewed as a product of PHS and SLS [15]. As SLS in string theory is interpreted as an invariance under a parity transformation in the transverse coordinates of D-branes, we can 
Class A (Boundary):

\begin{tabular}{c|cccccc|cccc|c|c}
\hline \hline & 0 & 1 & 2 & 3 & 4 & 5 & 6 & 7 & 8 & 9 & $d$ & $\mathrm{~A}$ \\
\hline D5 & $\times$ & $\times$ & & $\times$ & $\times$ & $\times$ & & & & $\times$ & & \\
\hline D5 & $\times$ & $\times$ & $\times$ & & & & $\times$ & $\times$ & $\times$ & & 1 & $\mathbb{Z}(1$ Weyl $)$ \\
D7 & $\times$ & $\times$ & $\times$ & $\times$ & $\times$ & & $\times$ & $\times$ & $\times$ & & 3 & $\mathbb{Z}(1$ Weyl $)$ \\
\hline \hline
\end{tabular}

Class AIII (Boundary):

\begin{tabular}{c|cccccc|cccc|c|c}
\hline \hline & 0 & 1 & 2 & 3 & 4 & 5 & 6 & 7 & 8 & 9 & $d$ & AIII \\
\hline D4 & $\times$ & & & $\times$ & $\times$ & $\times$ & & & & $\times$ & & \\
\hline D4 & $\times$ & & $\times$ & & & & $\times$ & $\times$ & $\times$ & & 0 & $\mathbb{Z}(2 \mathrm{Mj})$ \\
D6 & $\times$ & & $\times$ & $\times$ & $\times$ & & $\times$ & $\times$ & $\times$ & & 2 & $\mathbb{Z}(2 \mathrm{Mj})$ \\
\hline \hline
\end{tabular}

TABLE VI: Intersecting $\mathrm{D} p$-D $q$ systems realizing a boundary of class A and AIII TIs/TSCs.

interpret TRS as the multiplication of the complex conjugation and the parity transformation. This is precisely what is called the orientifold projection in string theory.

In this way, both TRS and PHS are related to the complex conjugation, and symmetry classes with either TRS or PHS (or both) thus belong to the real category from the K-theoretic viewpoint.

\section{A. Orientifold Projection and Basic Strategy}

Our strategy to find the D-brane configurations realizing TIs/TSCs in the eight real symmetry classes goes quite parallel to the complex case studied in the previous section. We consider cases with as minimal number of $\mathrm{DD}$ directions as possible, either $\# \mathrm{DD}=1$ or $\# \mathrm{DD}=2$, depending on the absence or presence of SLS, respectively. This is so since cases with \#DD $\geq 3$ are either unstable or lead to a system which can simply be viewed as copies of the one with \#DD $\geq 1,2$, as explained before.

The new ingredient in the real case is the presence of specific orientifolds. An orientifold projection is a $\mathbb{Z}_{2}$ action defined by the orientation reverse $\Omega$ followed by the parity transformation of $9-r$ coordinates $x_{i} \rightarrow-x_{i}$, where $r$ runs from -1 to 9 . The fixed point set of the $\mathbb{Z}_{2}$ action is a $(r+1)$-dimensional plane and called an orientifold $r$-plane (Or-plane). In superstring theory, there are two kinds of $\Omega$ projection: orthogonal (O) and symplectic $(\mathrm{Sp})$ types. They are distinguished by the different actions on the $N \times N$ matrix for $N$ coincident D-branes. Refer to 31] or the Appendix A of this paper for basics and especially to 38] for the analysis of orientifolds in unstable D-brane systems. Accordingly, there are two kinds of orientifold plane called $\mathrm{Or}^{-}-(\mathrm{O})$ and $\mathrm{O} r^{+}$-plane (Sp). When acting on the gauge fields on $N \mathrm{D} p$-branes parallel to the orientifold plane, the orientifold projection projects the original $\mathrm{U}(N)$ gauge group into the $\mathrm{SO}(N)$ and $\operatorname{Sp}(N)$ gauge group, respectively. The T-duality in the direction parallel to (orthogonal to) an $\mathrm{O} r^{ \pm}$-plane leads to an $\mathrm{O}(r-1)^{ \pm}\left[\mathrm{O}(r+1)^{ \pm}\right]$-plane, respectively.
Once we choose either $\mathrm{Or}^{-}$- or $\mathrm{O} r^{+}$-plane, we then specify $\mathrm{D} p$-brane. We assume that the $\mathrm{D} p$-brane has always an integer $\mathrm{K}$-theory topological charge. In other words, the $\mathrm{D} p$-brane preserves supersymmetry in the presence of $\mathrm{O} r$-plane, though it is broken in general when we add a D $q$-brane later. On the other hand, for the Dq-brane, we allow both supersymmetric and nonsupersymmetric configurations. The $\mathrm{D} q$-brane charge is interpreted as the topological charges of TIs/TSCs, which can be either $\mathbb{Z}$ or $\mathbb{Z}_{2}$, while the $\mathrm{D} p$-brane just provides an external gauge field which measures the topological charges (or Berry phases) induced by the Dq-brane. As opposed to the complex case, the ' $\mathrm{D} q$-brane' is not always the standard D $q$-brane with GSO projection of open strings.

As an example, consider D-branes in type IIB string with an $\mathrm{O}^{-}$-plane, i.e. type I string theory [24]. The D-brane charges are summarized in the Table III] The D1, D5 and D9-branes all have the integer charge $\mathbb{Z}$ and they are the standard BPS D-branes. On the other hand, $\mathrm{D}(-1)$ and $\mathrm{D} 7$-brane are $\mathbb{Z}_{2}$-charged and they are interpreted as a brane-antibrane system, i.e., $\mathrm{D}(-1)-\overline{\mathrm{D}}(-1)$ and D7-D 7 , respectively. An anti D $p$-brane $(\overline{\mathrm{D}} p)$ carries the opposite $\mathrm{RR}$ charge as compared with a $\mathrm{D} p$-brane and can be regarded as a higher dimensional generalization of anti-particle. Notice that brane-antibrane systems in type II string theory include tachyons in the open string spectrum [39]. However, in the presence of orientation projection $\Omega$, the tachyons are projected out and these brane-antibrane systems are stable. If we consider two copies of such a brane-antibrane system, then the tachyon appears from the open string which connects between two different systems [27. Therefore the D-brane charge is given by $\mathbb{Z}_{2}$. Moreover, one may notice the D0 and D8-brane are also $\mathbb{Z}_{2}$ charged. These are described by non-standard D-branes called non-BPS D-branes [40, which are defined by not requiring any GSO projections on the open strings attached on them. Note that only odd integer $p$ is allowed for $\mathrm{D} p$-branes in type IIB string theory. Again the tachyons which appear in the absence of the GSO projection, are eliminated by the $\Omega$ projection. Similar results can be understood for the $\mathrm{O}^{+}$by shifting $p \rightarrow p+4$ and the results for other values of $p$ can be obtained by the T-duality transformation.

We can realize all eight symmetry classes by considering $\mathrm{D} p-\mathrm{D} q$ systems in the presence of $\mathrm{O}^{ \pm}$or $\mathrm{O} 8^{ \pm}$[see Fig. 11 (b)] as we will explain below. Though it is also possible to proceed to the cases with $\mathrm{O} p^{ \pm}$-planes, in such systems we often encounter tachyonic instability due to the open strings between $\mathrm{D} p$ - and $\mathrm{D} q$-branes as we will discuss later.

\section{B. Class C and D}

First consider the case with $\mathrm{O}^{-}$or $\mathrm{O}^{+}$by requiring \#DD = 1, which guarantees the existence of gapless modes at the boundary. While only $\mathrm{O}^{-}$leads to su- 
persymmetric type I string theory, here we consider both because the T-dual of $\mathrm{O}^{+}$is equivalent to $\mathrm{O}^{+}$planes $(p \leq 8)$ in type II string theory. We can take $p=5$ again and can identify the number of $\mathrm{NN}$ directions as spacetime dimensions of TIs/TSCs, \#NN $=d+1$. This leads to the D5-D $q$ systems shown in Table VII. We can identify these configurations as class $\mathrm{C}$ and $\mathrm{D}$, which are characterized by the presence of PHS. Notice that these brane configurations are precisely the $\Omega$ projection of the previous class A brane configurations, and $\Omega$ projection directly corresponds to imposing PHS. Moreover, we can confirm that the D-brane charges agree with the topological charges of TIs/TSCs correctly, and that the fermion contents of these string theory realizations (denoted in the last two columns in Table VII either by " $n_{f}$ Mj" or " $n_{f}$ Di" with $n_{f}$ an integer) perfectly agree with the Dirac representative of TIs/TSCs constructed in Ref. [15]. The gauge group on D5- and D $q$-branes are also $\Omega$ projected and the result is summarized as in Table IV.

For example, let us consider the $d=2$ case. The class $\mathrm{C}$ and D TIs/TSCs are described by Chern-Simons theories with the $\mathrm{Sp}$ and $\mathrm{O}$ gauge groups, respectively. If we restrict to the case with the minimum number of $\mathrm{D} q$-branes, then the gauge group on the $\mathrm{D} 5$ - and $\mathrm{D} q$ branes are the same and it is $\mathrm{SU}(2)$ for class $\mathrm{C}$, while it is $\mathrm{O}(1)=\mathbb{Z}_{2}$ for class $\mathrm{D}$. We may view class $\mathrm{C}$ and $\mathrm{D}$ TIs/TSCs in $d=2$ as a close cousin of the QHE (class A), in a sense that the underlying topological field theories for these TIs/TSCs are the Chern-Simons theories with gauge group $\mathrm{Sp}, \mathrm{O}$, and $\mathrm{U}$, respectively.

\section{Class AII and AI}

In order to realize class AII and AI, we need to impose TRS but not PHS. As we have explained, TRS corresponds to the orientifold projection or equally the combination of the $\Omega$ projection and parity. Thus we introduce $\mathrm{O}^{ \pm}$instead of $\mathrm{O}^{ \pm}$and set $p=4$. By requiring $\# \mathrm{DD}=1$ as there is no SLS in AII or AI, we obtain the D-brane configurations as shown in Table VII (see also Fig.2 for class AII in $d=2$ ). As is clear from their Dbrane charge and the fermion spectrum, we can indeed identify these configurations with the class AII and AI. Though strictly speaking, the D-brane charges with an $\mathrm{O} p$-plane for $p \leq 8$ are classified by KR-theory, the same result can be obtained from KO-theory via T-duality for our purpose [35].

\section{Class CII, BDI, CI and DIII}

The other four classes possess SLS and thus we need to require $\# \mathrm{DD}=2$ for the brane constructions. Thus we can obtain their brane systems from the previous C, D, AII and AI brane setups by shifting \#DD in an obvious way. They are summarized in Table $\mathrm{VII}$ and again they reproduces the correct topological charges and the fermion spectra. In our convention, the SLS is imposed by requiring $x^{9}=0$ for all of these classes.

\section{E. Comments}

Even though the ten-dimensional string theories in the bulk are supersymmetric, our brane setups are not in general. When $\# \mathrm{ND}=4,8$ with $\mathbb{Z}$ charge, they exceptionally preserve a quarter of supersymmetries. In general, when $\# \mathrm{ND}=4$, there exist massive bosons in addition to the massive fermions. This happens when $d=4$ for class A, C, AI, AII, and when $d=3$ for class AIII, CII, CI and DIII. Since \#ND > 4 for all the other brane systems, we only have fermions from open strings between the $\mathrm{D} p$ - and $\mathrm{D} q$-branes and there are no tachyons.

In principle, we can take the T-duality further in $\mathrm{NN}$ directions. This generates $\mathrm{D} p-\mathrm{D} q$ systems in the presence of $\mathrm{O} r^{ \pm}$-plane for $r \leq 7$ as in Table VIII. This seems to lead to theories with different properties than the ten classes of TIs/TSCs. However, they have \#DD $\geq 3$ and as we explained before, such systems are either unstable because of open string tachyons, or essentially multiple copies of the ten classes which we already constructed from D-branes. Thus our D-brane construction reproduces precisely the ten fold classifications given in 15 .

Note also that we have succeeded to realize all TIs/TSCs in space dimensions $d \leq 4$, even though string theory lives in ten dimensions. This is so since we need to consider the common spacetime of $\mathrm{D} p$ - and $\mathrm{D} q$ branes. The existence of boundary gapless modes requires \#DD $\geq 1$ and the absence of tachyon argues $\# \mathrm{ND} \geq 4$. Thus we inevitably have the constraint

$$
d=\# \mathrm{NN}-1=9-\# \mathrm{DD}-\# \mathrm{ND} \leq 4 .
$$

\section{F. Field Theory Content}

We now describe the field theory content of our brane systems in more details and their link to condensed matter systems.

\section{1. primary series}

First, let us focus on the diagonal in Table If where lie TIs/TSCs characterized by an integer topological invariant. These TIs/TSCs can be called "primary series", since lower-dimensional TIs/TSCs characterized by a binary topological invariant can be derived from them by dimensional reduction. 8, 15] For $d=2 n$ dimensional TIs/TSCs, the relevant topological invariant is the Chern invariant defined for the (non-Abelian) Berry connection in momentum space, while for $d=2 n+1$ dimensional TIs/TSCs, the relevant topological invariant is the Chern-Simons invariant, and the winding number. 
Class C and D:

\begin{tabular}{c|cccccc|cccc|c|c|c}
\hline \hline & 0 & 1 & 2 & 3 & 4 & 5 & 6 & 7 & 8 & 9 & $d$ & $\mathrm{C}\left(\mathrm{O} 9^{-}\right)$ & $\mathrm{D}\left(\mathrm{O}^{+}\right)$ \\
\hline D5 & $\times$ & $\times$ & $\times$ & $\times$ & $\times$ & $\times$ & & & & & & \\
\hline D3 & $\times$ & & & & & & $\times$ & $\times$ & $\times$ & 0 & 0 & $\mathbb{Z}_{2}(2 \mathrm{Mj})$ \\
D4 & $\times$ & $\times$ & & & & & $\times$ & $\times$ & $\times$ & 1 & 0 & $\mathbb{Z}_{2}(1 \mathrm{Mj})$ \\
D5 & $\times$ & $\times$ & $\times$ & & & & $\times$ & $\times$ & $\times$ & 2 & $\mathbb{Z}(4 \mathrm{Mj})$ & $\mathbb{Z}(1 \mathrm{Mj})$ \\
D6 & $\times$ & $\times$ & $\times$ & $\times$ & & & $\times$ & $\times$ & $\times$ & 3 & 0 & 0 \\
D7 & $\times$ & $\times$ & $\times$ & $\times$ & $\times$ & $\times$ & $\times$ & $\times$ & 4 & $\mathbb{Z}_{2}(2 \mathrm{Di})$ & 0 \\
\hline \hline
\end{tabular}

Class CI and DIII:

\begin{tabular}{c|cccccc|cccc|c|c|c}
\hline \hline & 0 & 1 & 2 & 3 & 4 & 5 & 6 & 7 & 8 & 9 & $d$ & CI $\left(\mathrm{O}^{-}\right)$ & DIII $\left(\mathrm{O}^{+}\right)$ \\
\hline D5 & $\times$ & $\times$ & $\times$ & $\times$ & $\times$ & $\times$ & & & & & & \\
\hline D2 & $\times$ & & & & & & $\times$ & $\times$ & & 0 & 0 & 0 \\
D3 & $\times$ & $\times$ & & & & & $\times$ & $\times$ & & 1 & 0 & $\mathbb{Z}_{2}(2 \mathrm{Mj})$ \\
D4 & $\times$ & $\times$ & $\times$ & & & & $\times$ & $\times$ & & 2 & 0 & $\mathbb{Z}_{2}(2 \mathrm{Mj})$ \\
D5 & $\times$ & $\times$ & $\times$ & $\times$ & & $\times$ & $\times$ & & 3 & $\mathbb{Z}(4 \mathrm{Mj})$ & $\mathbb{Z}(1 \mathrm{Mj})$ \\
\hline \hline
\end{tabular}

Class AII and AI:

\begin{tabular}{c|ccccc|c|cccc|c|c|c}
\hline \hline & 0 & 1 & 2 & 3 & 4 & 5 & 6 & 7 & 8 & 9 & $d$ & $\mathrm{AII}\left(\mathrm{O} 8^{-}\right)$ & $\mathrm{AI}\left(\mathrm{O} 8^{+}\right)$ \\
\hline D4 & $\times$ & $\times$ & $\times$ & $\times$ & $\times$ & & & & & & & & \\
\hline D4 & $\times$ & & & & & & $\times$ & $\times$ & $\times$ & $\times$ & 0 & $\mathbb{Z}(4 \mathrm{Mj})$ & $\mathbb{Z}(1 \mathrm{Mj})$ \\
D5 & $\times$ & $\times$ & & & & & $\times$ & $\times$ & $\times$ & $\times$ & 1 & 0 & 0 \\
D6 & $\times$ & $\times$ & $\times$ & & & & $\times$ & $\times$ & $\times$ & $\times$ & 2 & $\mathbb{Z}_{2}(4 \mathrm{Mj})$ & 0 \\
D7 & $\times$ & $\times$ & $\times$ & $\times$ & & & $\times$ & $\times$ & $\times$ & $\times$ & 3 & $\mathbb{Z}_{2}(2 \mathrm{Mj})$ & 0 \\
D8 & $\times$ & $\times$ & $\times$ & $\times$ & $\times$ & & $\times$ & $\times$ & $\times$ & $\times$ & 4 & $\mathbb{Z}(1 \mathrm{Di})$ & $\mathbb{Z}(1 \mathrm{Di})$ \\
\hline \hline
\end{tabular}

Class CII and BDI:

\begin{tabular}{l|ccccc|c|cccc|c|c|c}
\hline \hline & 0 & 1 & 2 & 3 & 4 & 5 & 6 & 7 & 8 & 9 & $d$ & $\mathrm{CII}\left(\mathrm{O} 8^{-}\right)$ & $\mathrm{BDI}\left(\mathrm{O}^{+}\right)$ \\
\hline D4 & $\times$ & $\times$ & $\times$ & $\times$ & $\times$ & & & & & & & \\
\hline D3 & $\times$ & & & & & & $\times$ & $\times$ & $\times$ & 0 & 0 & $\mathbb{Z}_{2}(2 \mathrm{Mj})$ \\
D4 & $\times$ & $\times$ & & & & & $\times$ & $\times$ & $\times$ & 1 & $\mathbb{Z}(4 \mathrm{Mj})$ & $\mathbb{Z}(1 \mathrm{Mj})$ \\
D5 & $\times$ & $\times$ & $\times$ & & & & $\times$ & $\times$ & $\times$ & 2 & 0 & 0 \\
D6 & $\times$ & $\times$ & $\times$ & $\times$ & & $\times$ & $\times$ & $\times$ & 3 & $\mathbb{Z}_{2}(4 \mathrm{Mj})$ & 0 \\
D7 & $\times$ & $\times$ & $\times$ & $\times$ & $\times$ & $\times$ & $\times$ & $\times$ & 4 & $\mathbb{Z}_{2}(2 \mathrm{Di})$ & 0 \\
\hline \hline
\end{tabular}

TABLE VII: D $p$-D $q$ systems for eight "real" symmetry classes, where $p=5$ for classes C, D, CI, DIII, and $p=4$ for classes AII, AI, CII, BDI. For classes AII, AI, CII, BDI, the O8-plane extends except $x^{5}$ 37. In these tables, $\mathrm{D} p$ with $\mathbb{Z}_{2}$ charge denotes a $\mathrm{D} p-\overline{\mathrm{D}} p$ system for $p$ odd (or even) in type IIB (or IIA) string theory, a non-BPS D-brane for $p$ even (or odd) in type IIB (or IIA) string theory.

As we have been emphasizing, our string theory construction of TIs/TSCs naturally gives rise to gauge interactions, together with massive fermions. For the primary series, the internal gauge group is $\mathrm{O}(1)=\mathbb{Z}_{2}$. The $\mathbb{Z}_{2}$ gauge theory is somewhat difficult to formulate in the continuum spacetime - it is better put on a discretized spacetime (lattice). In string theory, the $\mathbb{Z}_{2}$ gauge group is visible when we consider a Wilson loop which is projected by the $\mathbb{Z}_{2}$ projection. The original Wilson loop takes continuous values of $U(1)$ but after the orientifold projection it only takes $\mathbb{Z}_{2}$ values \pm 1 .

The appearance of the $\mathbb{Z}_{2}$ gauge group is particularly indicative, since there exist interacting bosonic models defined on a lattice, which realize a topological phase that is described in terms of (emergent) fermions interacting with a $\mathbb{Z}_{2}$ gauge field. The microscopic model was first introduced by Kitaev 41] for $S=1 / 2$ spins on the honeycomb lattice, and can be exactly solvable. Since then, a huge number of generalizations/extensions of the original Kitaev model has been discussed. The original Kitaev model realizes, in the phase with broken TRS, a topological phase with non-Abelian statistics, which can be described in terms of emergent Majorana fermions in symmetry class $\mathrm{D}$ interacting with $\mathbb{Z}_{2}$ gauge field. Our string theory realization of the TI/TSC in class $\mathrm{D}$ in $d=2$ can be viewed as corresponding to the honeycomb lattice Kitaev model in the non-Abelian topological phase. A three-dimensional generalization of the honeycomb lattice Kitaev model to the diamond lattice was discussed in Ref. 42. As in the original Kitaev model, for a region of its phase diagram, the model realizes a topological phase which can be described in terms emergent Majorana fermions in symmetry class DIII interacting with $\mathbb{Z}_{2}$ gauge field. Again, our string theory realization of the TI/TSC in class DIII in $d=3$ can be viewed as corresponding to (a proper supersymmetric generalization of) this interacting bosonic model. (For AII $(d=4)$ and AI $(d=0)$, there is $1 / 4$ susy, and for DIII $(d=3)$, there is $1 / 8$ susy. For other TIs/SCs in the primary series, there is no susy.)

\section{2. first descendant}

Let us now focus on TIs/TSCs with binary $\mathbb{Z}_{2}$ topological invariant lying beneath/immediate left to the primary series in Table If i.e, BDI $(d=0)$, D $(d=1)$, DIII $(d=2)$, and AII $(d=3)$. These TIs/TSCs can be obtained from the primary series by dimensional reduction, and therefore can be called the first descendant. The internal gauge group is $\mathrm{O}(1)=\mathbb{Z}_{2}$ (Table $[\mathrm{IV})$. In our string theory realization of TIs/TSCs, the first descendant is realized by a D-brane with $\mathbb{Z}_{2} \mathrm{~K}$-theory charge, which is called a non-BPS D-brane [40].

a. the $\mathbb{Z}_{2}$ topological insulator (class AII in $d=3$ ) Among the $\mathbb{Z}_{2}$ TIs/TSCs of the first descendent is the $d=$ 3 -dimensional $\mathbb{Z}_{2}$ topological insulator in class AII which is of most interest recently. The external gauge group for this case is $G=\mathrm{Sp}(1)=\mathrm{SU}(2)$ (Table [V). By moving the $\mathrm{D} p$-brane away from O8-plane, this can further be broken into its diagonal subgroup U(1), which couples to fermions as an external "electromagnetic" U(1) gauge field. In this case, after integrating out the fermions, the $\theta$ term at $\theta=\pi$,

$$
\frac{m}{16 \pi|m|} \int_{\mathbb{R}^{1,3}} F \wedge F
$$

will be induced, in agreement with Refs. 8, 43]. 


\section{3. second descendant}

The $\mathbb{Z}_{2}$ TIs/TSCs lying beneath/immediate left to the first descendent in Table If, i.e, D $(d=0)$, DIII $(d=1)$, AII $(d=2)$, and CII $(d=3)$, can be called the second descendant, since they can be obtained by dimensionally reducing the primary series twice. For these $\mathbb{Z}_{2}$ TIs/TSCs the internal gauge group is U(1). In our string theory realization of TIs/TSCs, the second descendant is realized by a D-brane with a $\mathbb{Z}_{2} \mathrm{~K}$-theory charge, which is a bound state of a D-brane and a anti D-brane (so called brane-antibrane system) [39].

For class D and DIII, the U(1) gauge field couples to two real fermions which can be combined into a single complex field. For class AII and CII, the fermion spectrum consists of $4 \mathrm{Mj}$. In this case, the external gauge field is $\operatorname{Sp}(1)=\mathrm{SU}(2)$, which couples to a doublet, $\psi_{\uparrow / \downarrow}$. Each has $2 \mathrm{Mj}$ (=1Di) degrees of freedom and couples to a $\mathrm{U}(1)$ internal gauge field.

a. the quantum spin Hall effect (class AII in $d=2$ ) Among $\mathbb{Z}_{2}$ TIs/TSCs in the second descendants, we now take a closer look at a TI in class AII in $d=2$, which is the QSHE described in Fig. 2. The low-energy effective theory of the $\mathrm{D} p-\mathrm{D} q$ system is summarized by the following effective Lagrangian

$$
\mathcal{L}=\bar{\psi}\left[\gamma^{\mu}\left(i \partial_{\mu}-A_{\mu}-\tilde{A}_{\mu}\right)-m M\right] \psi+\cdots
$$

where $\psi=\left(\psi_{\uparrow}, \psi_{\downarrow}\right)^{T}$, and $M$ is a diagonal mass matrix whose eigenvalue is \pm 1 for $\psi_{\uparrow / \downarrow}$, respectively. The gamma matrices and mass matrices are given, in terms of two sets of the $2 \times 2$ Pauli matrices $\sigma_{1,2,3}$ and $\tau_{1,2,3}$ by $\gamma^{\mu=0,1,2}=\sigma_{3} \otimes \tau_{0}, i \sigma_{2} \otimes \tau_{0},-i \sigma_{1} \otimes \tau_{0}$, and $M=\sigma_{0} \otimes \tau_{3}$, say. The $4 \times 4$ Dirac Hamiltonian of the same type has been widely discussed as a model of the QSHE [1, 4] The edge mode of the QSHE consists of odd number of Kramers pairs, which is just one for the Dirac model (10).

The gauge group of this theory is $G \times \tilde{G}=\mathrm{SU}(2) \times \mathrm{U}(1)$, i.e., the gauge field $A_{\mu}$ on the $\mathrm{D} p$-brane is $\mathrm{SU}(2)$, while $\tilde{A}_{\mu}$ on the $\mathrm{D} q$-brane is $\mathrm{U}(1)$. The $\mathrm{U}(1)$ gauge field can be interpreted as the usual electric-magnetic field, while the $\mathrm{SU}(2)$ can be viewed as a (fictitious) gauge field which couples to the $\mathrm{SU}(2)$ spin. Below we only consider the third component (proportional to $\tau_{3}$ ) of the $\mathrm{SU}(2)$ gauge field $A_{\mu}^{3}$. By integrating out the bi-fundamental fermions $\psi$, we find the double Chern-Simons action $\left(A_{\mu}=A_{\mu}^{3}\right)$,

$$
\begin{aligned}
S_{\mathrm{dCS}}= & \frac{1}{8 \pi} \int(A+\tilde{A}) \wedge d(A+\tilde{A}) \\
& \quad-\frac{1}{8 \pi} \int(A-\tilde{A}) \wedge d(A-\tilde{A}) \\
= & \frac{1}{2 \pi} \int A \wedge d \tilde{A} .
\end{aligned}
$$

\begin{tabular}{|c|c|c|c|c|c|}
\hline \#DD & $\left(\mathrm{O} 9^{-}, \mathrm{O} 9^{+}\right.$ & $\left(\mathrm{O}^{-}, \mathrm{O}^{+}\right)$ & $\left(\mathrm{O} 7^{-}, \mathrm{O} 7^{+}\right)$ & $\mathrm{O}^{-}, \mathrm{O}^{+}$ & $\left.\mathrm{O} 5^{-}, \mathrm{O} 5^{+}\right)$ \\
\hline 0 & Chiral & & & & \\
\hline 1 & $(\mathrm{C}, \mathrm{D})$ & (AII,AI) & & & \\
\hline 2 & (CI,DIII) & (CII,BDI) & (DIII,CI) & & \\
\hline 3 & $d \leq 2$ & $d \leq 2$ & $d \leq 2$ & $d \leq 2$ & \\
\hline 4 & $d \leq 1$ & $d \leq 1$ & $d \leq 1$ & $d \leq 1$ & $d \leq 1$ \\
\hline
\end{tabular}

From the effective action we can find the QSHE,

$$
j_{\text {spin }}^{\mu}=\frac{1}{2} \frac{\delta S_{\mathrm{dCS}}}{\delta A_{\mu}}=\frac{1}{4 \pi} \epsilon^{\mu \nu \rho} \partial_{\nu} \tilde{A}_{\rho},
$$

TABLE VIII: $\mathrm{D} p$-D $q$ systems in the presence of an O-plane; "Tac" represents the presence of tachyons in open strings between $\mathrm{D} p$ and $\mathrm{D} q$ at least when $d=3$; " $d \geq 2$ " means the constraint of possible spatial dimensions $d$ in the brane systems. This is due to either an absence of corresponding brane systems or tachyonic instability. "Chiral" denotes the existence of chiral fermions and is interpreted as boundary (edge) states. The horizontal direction is shifted by a T-duality in the NN direction.

Class AII:

\begin{tabular}{c|cccccc|cccc|cc}
\hline \hline & 0 & 1 & 2 & 3 & 4 & 5 & 6 & 7 & 8 & 9 & $d$ & $\mathrm{AII}$ \\
\hline $\mathrm{O}^{-}$ & $\times$ & $\times$ & $\times$ & $\times$ & & & & & & & \\
$\mathrm{D} 7(=\mathrm{D} p)$ & $\times$ & $\times$ & $\times$ & $\times$ & & $\times$ & $\times$ & $\times$ & $\times$ & & \\
\hline $\mathrm{D} 1$ & $\times$ & \multicolumn{1}{c|}{$\times 1$} & & & & & & 0 & $\mathbb{Z}(4 \mathrm{Mj})$ \\
$\mathrm{D} 2$ & $\times$ & $\times$ & & & $\times$ & & & & & 1 & \\
D3 & $\times$ & $\times$ & $\times$ & & $\times$ & & & & & 2 & $\mathbb{Z}_{2}(4 \mathrm{Mj})$ \\
D4 & $\times$ & $\times$ & $\times$ & $\times$ & $\times$ & & & & 3 & $\mathbb{Z}_{2}(2 \mathrm{Mj})$ \\
\hline \hline
\end{tabular}

Class AII (Boundary):

\begin{tabular}{c|cccccc|cccc|c|c}
\hline \hline & 0 & 1 & 2 & 3 & 4 & 5 & 6 & 7 & 8 & 9 & $d$ & AII \\
\hline O33 $^{-}$ & $\times$ & $\times$ & $\times$ & $\times$ & & & & & & & & \\
Edge D7 & $\times$ & & $\times$ & $\times$ & & $\times$ & $\times$ & $\times$ & $\times$ & $\times$ & & \\
\hline D3 & $\times$ & $\times$ & $\times$ & & $\times$ & & & & & & 1 & $\mathbb{Z}_{2}(2 \mathrm{Mj})$ \\
D4 & $\times$ & $\times$ & $\times$ & $\times$ & $\times$ & & & & & & 2 & $\mathbb{Z}_{2}(2 \mathrm{Mj})$ \\
\hline \hline
\end{tabular}

TABLE IX: D-brane configurations for class AII TIs in a T-dualized setup (top) and the brane construction of their boundary gapless modes (bottom). Here, D3 and D4 express a D3- $\bar{D} 3$ system and a non-BPS D4-brane, respectively.

where the factor $1 / 2$ comes from the basic fact that the spin of an electron is $\hbar / 2$. (See for example, Refs. 44, 45.)

\section{TIs/TSCs with $2 \mathbb{Z}$ topological charge}

Finally, for TIs/TSCs labeled by $2 \mathbb{Z}$ in Table $\mathbb{E}$, i.e., AII $(d=0)$, CII $(d=1), \mathrm{C}(d=2)$, and CI $(d=3)$, the gauge group is $G \times \tilde{G}=\mathrm{SU}(2) \times \mathrm{SU}(2)$, with $4 \mathrm{Mj}$ fermions in bi-fundamental.

An interesting example of TSC in class $\mathrm{C}(d=2)$ is the $d+i d$-wave SC, which is spin singlet with broken TRS 46, 47. A lattice model of three-dimensional topological $\mathrm{SC}$ in class CI, which is spin singlet and invariant under time-reversal, was constructed in Ref. [22]. 


\section{G. Boundary Gapless Modes of TIs/TSCs}

As in the complex case, boundary modes of the real case can be realized as an intersecting $\mathrm{D} p-\mathrm{D} q$ system. Here let us focus on class AII. For convenience, we take T-duality of the setup in Table VII in five ND directions, and consider the setup in the first table in IX], whose sketch is depicted in Fig. 2. Intersecting $\mathrm{D} p$-D $q$ systems describing the boundary of class AII TIs can then be obtained by exchanging $x^{1}$ and $x^{9}$ for the D7-brane, as in the second table in IX.

The analysis on the open string spectrum shows that the edge mode in $d=2$, i.e. the QSHE, is not chiral as opposed to class A TIs as expected. Indeed, the Dqbrane is now given by the D3- $\overline{\mathrm{D}} 3$ brane-anti-brane system (see Sec. IIIA) and its intersection with the bent D7brane leads to left and right-moving fermion on the $1+1$ dimensional boundary (see Fig. 3).

We can also see that the edge state configurations shown in Table IX are actually unstable because the D7brane bent toward the O3-plane is reflected back as an anti D7-brane at the O-plane. Since we inevitably have a parallel D7- and $\bar{D} 7$-brane at the same time, this system has in general tachyons and is unstable unless we fine tune its intersection angle. This actually is nothing but requiring TRS, since the open string tachyon field $T$ is odd under time-reversal. This can be regarded as an example where the presence of interactions non-trivially change stabilities of topological insulators. A similar instability occurs for class AI, CII and BDI.

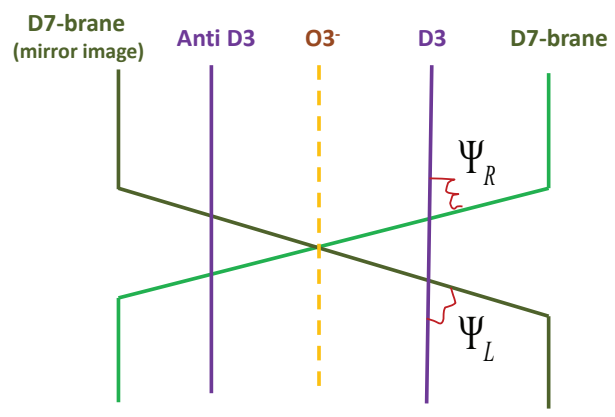

FIG. 3: The brane configuration of the edge state of the QSHE (class AII in $d=2$ ).

\section{CONCLUSIONS}

The tenfold classification of TIs/SCs was derived previously by several different approaches. One of the reasonings in Ref. 15] that leads to the classification is the socalled boundary-to-bulk approach; i.e,. exhaustive study of non-Anderson localized state at boundaries which signals the existence of the topological bulk theory. On the other hand, Kitaev proposed K-theory approach for the classification. Our string theory realization of TIs/TSCs can be thought of yet another derivation of the tenfold classification. It is quite compelling that all different approaches give the identical table.

It is worth while mentioning that the construction of TIs/TSCs from $\mathrm{D} p$ - $\mathrm{D} q$ systems is highly constrained in the following sense: $\mathrm{D} p-\mathrm{D} q$ systems which do not correspond to a bulk TI/TSC or a boundary of a TI/TSC (i.e., an intersecting $\mathrm{D} p$-D $q$ system) suffer from the existence of tachyons in the open string spectrum or are just multiple copies of the minimal fermion systems for the ten classes.

It is also interesting to note that, for the Dirac representative of TIs/TSCs which are constructed systematically in Ref. [15], the momentum dependence of the projection operator of Bloch wavefunctions, which is one of key ingredients in the classification of TIs/TSCs, looks quite similar to the spatial profile of the tachyon field in string theory in real space 24, 25.

One of the implications of our D-brane construction of TIs/TSCs is the underlying field theory description. When a charge of some sort is conserved, one can describe the topological phase in terms of effective field theory for the linear responses, but this is not always the case. For example, the $d=2$-dimensional p-wave SC is one of representative and most important topological phases in $d=2$, but its description in terms of field theory is not yet clearly understood, partly because there is no conserved quantum number. An effective (topological) field theory description, if exists, suggests the topological phase in question is robust against several perturbations, in particular against interactions. D-branes not only carry a K-theory charge which is necessary to describe topological phases, but they also come with the WZ term. This sheds a light on field theory description of TIs/TSCs in all symmetry classes.

Finally, to put our D-brane construction in a perspective, we can consider holographic descriptions of these systems by extending the constructions in 33, 34] in principle, though it is not possible to take the large- $N$ limit of $\mathbb{Z}_{2}$ charged D-branes. For a closely related holographic construction of fractional topological insulators, refer to the recent paper 48]. It would be interesting to holographically calculate the entanglement entropy 49] as it is expected to distinguish various topological phases, including topological insulators/superconductors [50].

\section{Acknowledgments}

We acknowledge "Quantum Criticality and the AdS/CFT correspondence" miniprogram at KITP, "Quantum Theory and Symmetries 6" conference at University of Kentucky. We would like to thank K. Hori, A. Karch, A. Kitaev, P. Kraus, A. Ludwig, J. Moore, M. Oshikawa, V. Schomerus, and S. Sugimoto for useful discussion, and A. Furusaki for his clear lecture at "De- 
velopment of Quantum Field Theory and String Theory" (YITP-W-09-04) at Kyoto University. SR thanks Center for Condensed Matter Theory at University of California, Berkeley for its support. TT is supported in part by JSPS Grant-in-Aid for Scientific Research No. 20740132, and by JSPS Grant-in-Aid for Creative Scientific Research No. 19GS0219.

\section{Appendix A: Overview on the Calculations of Open String Spectra}

In this appendix, we give an overview on how to calculate the fermion spectrum (the number of fermion species) arising from open strings between $\mathrm{D} p$ - and $\mathrm{D} q$ branes. We neglect all stringy modes and focus on the low energy limit. For more details, refer to textbooks, e.g., Ref. [31].

\section{D-branes in Type II String Theory (Complex Case)}

We first consider unoriented strings and do not impose the orientifold projection. If we set $\# \mathrm{ND}=2 k$, then the number $n_{F}$ of independent components of on-shell complex fermions in the $\mathrm{D} p$ - $\mathrm{D} q$ system is given by

$$
n_{F}=2^{5-k} \times \frac{1}{2} \times \frac{1}{2}=2^{3-k},
$$

where each of the three factors describe the degeneracy of the fermion zero modes in NN and DD directions, the GSO projection, and the Dirac equation constraint, respectively. The mass of these fermions are proportional to the distance between the $\mathrm{D} p$ - and $\mathrm{D} q$-branes. Notice that this counting has already taken into account the two orientations of the open string. For example, for the D5D5 system with $k=3$, we find $n_{F}=1$, i.e. one complex fermion in $2+1$ dimensions. If we consider the D1-D9 system, we have $k=4$ and thus find $n_{F}=1 / 2$, which means one chiral left-moving fermion in $1+1$ dimensions.

In the case where \#ND $=2 k-1$, the D $q$-brane is a nonBPS brane 40, which is defined by a D-brane without the GSO projection. We thus get

$$
n_{F}=2^{5-k} \times \frac{1}{2}=2^{4-k}
$$

\section{Orientifold Projections (Real Case)}

We now consider oriented strings to describe the real case, and take into account the orientifold projection (denoted by $\Omega$ ). The $\Omega$ projection in the csae of the O9-plane acts on $\mathrm{D} p$-branes as follows

$$
\begin{array}{ll}
\Omega|\mathrm{D} p\rangle=|\overline{\mathrm{D} p}\rangle, & p=-1,3,7 \\
\Omega|\mathrm{D} p\rangle=|\mathrm{D} p\rangle, \quad p \in \text { others }
\end{array}
$$

where $|\mathrm{D} p\rangle$ and $|\overline{\mathrm{D} p}\rangle$ are the boundary states of $\mathrm{D} p$-brane and anti $\mathrm{D} p$-brane, which express the D-brane states in the closed string Hilbert space. This is simply because in type I string theory, the 0 and 4 -form RR fields are projected out. In order to understand the action of $\Omega$ on open string spectrum, we need to introduce the action of $\Omega$ on the Chan-Paton factor. There are two possible actions: SO (called $\mathrm{O}^{-}{ }^{-}$-plane) and $\mathrm{Sp}$ (called $\mathrm{O}^{+}{ }^{+}$-plane) type. Each of them is defined by the action on the ChanPaton matrix $\Lambda$ :

$$
\Lambda \rightarrow \gamma \Lambda^{T} \gamma^{-1}
$$

with

$$
\gamma_{\mathrm{SO}}=1, \quad \gamma_{\mathrm{Sp}}=\sigma_{2} \otimes 1
$$

In addition we need to take into account (A3). As clear from the definition, the dimension of the Chan-Paton matrix for the Sp projection has to be even.

For example, for a D1-brane, the massless bosonic modes (the gauge field $A_{\mu}$ and the transverse scalars $\phi^{i}$ ) obey the following projection in the presence of $09^{-}$plane:

$$
A_{\mu}^{T}=-A_{\mu}, \quad\left(\phi^{i}\right)^{T}=\phi^{i},
$$

where the minus sign in front of the gauge field is due to the $\Omega$ action on the world-sheet oscillators. This leads to the gauge group $\mathrm{SO}(N)$. On the other hand, in the presence of $\mathrm{O} 9^{+}$-plane, we have the projection

$$
\gamma_{\mathrm{Sp}} A_{\mu}^{T} \gamma_{\mathrm{Sp}}=-A_{\mu}, \quad \gamma_{\mathrm{Sp}} \phi_{i}^{T} \gamma_{\mathrm{Sp}}=\phi_{i}
$$

from which we obtain the gauge group $\operatorname{Sp}(N)$.

On the other hand, if we are interested in the open string between two different $D$-branes, then the $\Omega$ projection just relates the two open strings extending opposite directions. If we define $\Psi_{12}$ as a field from such a open string, this projection is explicitly given as follows

$$
\gamma_{1} \Psi_{12}^{T} \gamma_{2}^{-1}=a \Psi_{21}
$$

where $a$ can be \pm 1 or $\pm i$. Thus this just reduces the original degrees of freedom by half.

Let us now consider the fermion spectra in the D5-D $q$ system. To find the spectrum, it is important to note that the non-BPS D-branes do not have any GSO projection and lead to Majorana instead of Weyl fermions. Also we should note that the D5-D3 system with $09^{+}$-plane (class D) and the D5-D7 system with $\mathrm{O}^{-}$-plane (class C) should be understood as the $\Omega$ projection of the D5D $3-\overline{\mathrm{D} 3}$ and D5 - D7 - $\overline{\mathrm{D} 7}$ system in type IIB string. Another important fact is that there are two D5-branes coincident in the case of $\mathrm{O}^{-}$-plane and so we have to double the spectrum in this case. These considerations lead to the fermion spectra summarized in Table $\mathrm{V}$ and Table VII. 
[1] C. L. Kane and E. J. Mele, Phys. Rev. Lett. 95, 146802 (2005); 95, 226801 (2005).

[2] R. Roy, Phys. Rev. B 79, 195321 (2009); ibid, 195322 (2009).

[3] J. E. Moore and L. Balents, Phys. Rev. B 75, 121306(R) (2007).

[4] B. A. Bernevig, T. L. Hughes, and S.-C. Zhang, Science 314, 1757 (2006).

[5] M. König et al., Science 318, 766 (2007).

[6] L. Fu, C. L. Kane, and E. J. Mele, Phys. Rev. Lett. 98, 106803 (2007).

[7] L. Fu and C. L. Kane, Phys. Rev. B 76, 045302 (2007).

[8] X.-L. Qi, T. Hughes, and S.-C. Zhang, Phys. Rev. B 78, 195424 (2008).

[9] D. Hsieh et al, Nature 452, 970 (2008).

[10] D. Hsieh, Science 323, 919 (2009).

[11] Y. Xia et al, Nature Phys. 5, 398 (2009).

[12] D. Hsieh et al, Nature 460, 1101 (2009).

[13] Y. L. Chen et al, Science 325, 178 (2009).

[14] M. Z. Hasan, and C. L. Kane, arXiv: 1002.3895.

[15] A. Schnyder, S. Ryu, A. Furusaki, A. Ludwig, Phys. Rev. B 78, 195125 (2008); AIP Conf. Proc. 1134, 10 (2009); S. Ryu, A. Schnyder, A. Furusaki, and A. Ludwig, New J. Phys. 12065010 (2010).

[16] A. Kitaev, AIP Conf. Proc. 1134, 22 (2009).

[17] For an application of K-theory to gapless fermion systems with a fermi surface with the parallel between K-theory classification of D-brane charges, see P. Horava, Phys. Rev. Lett. 95, 016405 (2005).

[18] M. R. Zirnbauer, J. Math. Phys. 37, 4986 (1996).

[19] A. Altland and M. R. Zirnbauer, Phys. Rev. B 55, 1142 (1997).

[20] See, e.g., also a more recent discussion: P. Heinzner, A. Huckleberry, and M. R. Zirnbauer, Commun. Math. Phys. 257, 725 (2005).

[21] S. Helgason, "Differential geometry, Lie groups and symmetric spaces", Academic Press, New York, (1978).

[22] A. P. Schnyder, S. Ryu, and A. W. W. Ludwig, Phys. Rev. Lett. 102, 196804 (2009).

[23] See for example, S. -S. Lee, S. Ryu, Phys. Rev. Lett. 100, 186807 (2008); M. W. Young, S. -S. Lee, C. Kallin, Phys. Rev. B 78, 125316 (2008); L. Fidkowski, and A. Kitaev, arXiv:0904.2197; M. Levin and A. Stern, Phys. Rev. Lett. 103, 196803 (2009); D. Pesin, and L. Balents, Nature Phys. 6, 376 (2010); S. Rachel, and K. Le Hur, 1003.2238; J. Maciejko, X. -L. Qi, A. Karch, S. C. Zhang, arXiv:1004.3628; B. Swingle, M. Barkeshli, J. McGreevy, T. Senthil, arXiv:1005.1076; J. Wen, A. Ruegg, C. -C. Joseph Wang, G. A. Fiete, 1005.4061; C. N. Varney, K. Sun, M. Rigol, V. Galitski, 1007.3502.

[24] E. Witten, JHEP 9812, 019 (1998).

[25] P. Horava, Adv. Theor. Math. Phys. 2 (1999) 1373.
[26] A. Sen, Int. J. Mod. Phys. A 20, 5513 (2005).

[27] A. Sen, JHEP 9809, 023 (1998) [arXiv:hep-th/9808141].

[28] For a different approach to TIs from string theory, see, for example, A. Karch, Phys. Rev. Lett. 103, 171601 (2009).

[29] X. -G. Wen, Mod. Phys. Lett. B 5, 39 (1991); Phys. Rev. B 60, 8827 (1999).

[30] S. Ryu and T. Takayanagi, arXiv:1001.0763 (2010).

[31] J. Polchinski, "String theory. Vol. 1 and 2", Cambridge, UK: Univ. Pr. (1998).

[32] S. J. Rey, Prog. Theor. Phys. 177, 128 (2009).

[33] J. L. Davis, P. Kraus and A. Shah, JHEP 0811, 020 (2008); O. Bergman, N. Jokela, G. Lifschytz and M. Lippert, arXiv:1003.4965 [hep-th].

[34] M. Fujita, W. Li, S. Ryu, and T. Takayanagi, JHEP 0906, 066 (2009); Y. Hikida, W. Li and T. Takayanagi, JHEP 0907 (2009) 065 .

[35] For a KR theory analysis in similar brane systems, see T. Imoto, T. Sakai and S. Sugimoto, arXiv:0907.2968, and references therein.

[36] Pavan Hosur, Shinsei Ryu, and Ashvin Vishwanath, Phys. Rev. B, 81, 045120 (2010).

[37] For $d=0$, what we mean by Majorana ("Mj") is a fermion with some real condition.

[38] O. Bergman, "Tachyon condensation in unstable type I D-brane systems," JHEP 0011 (2000) 015 [arXiv:hepth/0009252].

[39] A. Sen, JHEP 9808, 012 (1998) [arXiv:hep-th/9805170].

[40] O. Bergman and M. R. Gaberdiel, "Stable non-BPS Dparticles," Phys. Lett. B 441, 133 (1998) [arXiv:hepth/9806155].

[41] Alexei Kitaev, Ann. of Phys. 321, 2 (2006).

[42] Shinsei Ryu, Phys. Rev. B 79, 075124 (2009).

[43] A. M. Essin, J. E. Moore and D. Vanderbilt, Phys. Rev. Lett. 102, 146805 (2009).

[44] B. Andrei Bernevig, Shou-Cheng Zhang, Phys. Rev. Lett. 96, 106802 (2006).

[45] Shinsei Ryu, Christopher Mudry, Chang-Yu Hou, and Claudio Chamon Phys. Rev. B 80, 205319 (2009).

[46] N. Read and D. Green, Phys. Rev. B 61, 10267 (2000).

[47] T. Senthil, J. B. Marston, and M. P. A. Fisher, Phys. Rev. B 60, 4245 (1999); I. A. Gruzberg, A. W. W. Ludwig, and N. Read Phys. Rev. Lett. 82, 4524 (1999).

[48] Carlos Hoyos-Badajoz, Kristan Jensen, Andreas Karch, arXiv: 1005.3253.

[49] S. Ryu and T. Takayanagi, Phys. Rev. Lett. 96 (2006) 181602; JHEP 0608 (2006) 045; T. Nishioka, S. Ryu and T. Takayanagi, J. Phys. A 42 (2009) 504008.

[50] A. Kitaev and J. Preskill, Phys. Rev. Lett. 96, 110404 (2006); M. Levin and X-G. Wen, Phys. Rev. Lett. 96, 110405 (2006). 